\title{
Optical and Microwave Detection of Wave Breaking in the Surf Zone
}

\author{
Patricio A. Catalán, Merrick C. Haller, Robert A. Holman, and William J. Plant, Member, IEEE
}

\begin{abstract}
Synchronous and colocated optical and microwave signals from waves in the surf zone are presented and analyzed. The field data were collected using a high-resolution video system and a calibrated horizontally polarized marine radar during the decaying phase of a storm. The resulting changes in the received signals from varying environmental conditions were analyzed. The analysis of the optical signal histograms showed functional shapes that were in accordance with the expected imaging mechanisms from the breaking and nonbreaking waves. For the microwave returns, the histogram shape showed a little dependence on the environmental parameters and exhibited an inflexion point at high returned power that is attributed to a change in the scattering mechanism. The high intensity signals were clearly associated with active wave breaking. However, with either sensor, it can be difficult to effectively isolate the wave breaking signature from other sources, such as a remnant foam or the highly steepened nonbreaking waves. A combined method was developed using the joint histograms from both sensors, and it is shown to effectively discriminate between active breaking, remnant foam, and steepened waves. The new separation method allows a further analysis of the microwave scattering from the breaking waves and a better quantification of the length scales of the breaking wave roller and the spatial/temporal distribution of wave breaking and wave dissipation in the surf zone.
\end{abstract}

Index Terms-Nearshore, optical imaging, radar, remote sensing, sea surface, surface waves, wave breaking.

\section{INTRODUCTION}

$\mathbf{O}$ BSERVATIONS of the location and frequency of the occurrence of wave breaking are important in improving our understanding and predictive capabilities of nearshore hydrodynamics. Wave breaking leads to a transfer of momentum that is the dominant driver of surfzone currents and is also important for the resuspension and transport of sediments. The wave breaking roller is the physical structure generated in the breaking process, and it consists of a turbulent body of air and

Manuscript received June 2, 2010; revised October 26, 2010; accepted November 16, 2010. Date of publication January 5, 2011; date of current version May 20, 2011. This work was supported in part by the Office of Naval Research under award numbers N00014-06-1-0317, N00014-08-1-0636, and N00014-07-1-0490 and in part by FONDECYT under award number 11090201.

P. A. Catalán is with the Departmento de Obras Civiles, Universidad Santa María, Valparaíso 110-V, Chile (e-mail: patricio.catalan@usm.cl).

M. C. Haller is with the School of Civil and Construction Engineering, Oregon State University, Corvallis, OR 97331 USA.

R. A. Holman is with the College of Oceanic and Atmospheric Sciences, Oregon State University, Corvallis, OR 97331 USA.

W. J. Plant is with the Applied Physics Laboratory, University of Washington, Seattle, WA 98105 USA.

Digital Object Identifier 10.1109/TGRS.2010.2095864 water that develops on and propagates with the front face of a surfzone wave. The roller can be a dominant factor in the surfzone mass balance and in the generation of cross-shore currents [1]. In general, wave breaking exhibits a significant spatial and temporal variability that is difficult to observe and accurately predict in detail.

For example, models for nearshore hydrodynamics require a description at some level of wave breaking quantities, such as the onset of breaking, the fraction of the breaking waves, and the probability distribution of the breaking wave heights. These quantities appear in wave models of all types-parametric wave evolution models [2]-[4], third generation spectral models [5], or models that are based on the mild slope equation [6], and they are needed in order to quantify the wave dissipation and to calculate the additional quantities of the momentum transfer. In addition, other work has shown that the physical scales of the wave roller are directly related to dissipation [7], [8]. Therefore, the observations of the wave roller physical scales and the spatial/temporal distribution of wave breaking will support improvements in both our understanding of wave breaking statistics and the relationship between roller-related quantities and dissipation models.

However, the proper identification of wave breaking is a difficult task. Somewhat loosely defined as the transformation of organized wave energy into other energy states (turbulence, heat, and sound), wave breaking is not simply quantifiable. Some single-point methods exist [9], but the synoptic remote sensing approaches are best suited in identifying the space/time variability of the wave breaking occurrence. Furthermore, wave breaking signatures are usually very prominent in a number of different remote sensing modalities. Some examples are the following: acoustic [10], [11], optical [12], [13], infrared [14], [15], and microwave [16], [17]. Nonetheless, other phenomena can still contaminate the signal, causing difficulties in the accurate discrimination of breaking from other phenomena. For example, in the surf zone, the locations of preferential breaking are correlated with the increased mean intensity values of a series of optical images [18] or microwave images [19], [20], but these results can be affected by the presence of persistent foam or steepening waves, respectively.

Time exposures or the map of the temporal mean of the signal corresponds to the most basic statistical measure that can be used to discriminate between the breaking and nonbreaking waves. However, the probability density functions (pdfs) and the cumulative density functions of the time-varying signals encapsulate a higher level of information. The pdf approach has been used, for instance, in microwave-based maritime surveillance and target detection [21], [22] but only recently with 
optical data [23]. In addition, there is some previous work examining simultaneous signals in optical and microwave sensors [17], [24], [25]. However, the procedures for wave breaking identification have been fairly qualitative, and a high level of uncertainty persists. In the present work, our objective is to improve our understanding of both the optical and microwave signals arising from different sources in the surf zone, with the overarching goal of obtaining improved identification and measurements of breaking events on a wave-by-wave basis.

\section{IMAGing OF THE OCEAN Surface}

In the open ocean, wave breaking takes place over a wide range of temporal and spatial scales: from individual microbreaking events that produce small amounts of bubbles, turbulence, and spray but do not produce a strong optical signature (i.e., turbulent whitewater or foam) to larger scale events that are optically bright. In the following, we define these larger events as the signal of interest, and their imaging characteristics will be sensor dependent. We focus here on the occurrence and identification of these large events because they are a dominant process in surfzone hydrodynamics.

\section{A. Optical}

Optical sensing systems measure the radiance reaching the sensor at wavelengths in the visible band. The source is the sun irradiance that is being reflected by the ocean surface after being absorbed and diffused by the atmosphere, while the upwelling and direct sun radiances are often neglected. For the nonbreaking waves, the first approximation assumes the sky radiance to be isotropic and homogeneous, which leads to the observed radiance that is being dependent solely on the Fresnel reflection coefficient $R$. $R$, in turn, can be related to the surface slope of the ocean $s$. The relationship between $R$ and the surface slope is nonlinear, and it exhibits a strong asymmetry between positive (surface normal pointing toward the camera) and negative slopes. The latter has a stronger dependence, which means that small variations in negative slope yield significant variations in $R$ and, therefore, the radiant intensity [26]. However, if the slopes are small $\left(s<20^{\circ}\right)$, the relationship can be treated as linear [27].

The breaking waves depart from this specular reflection model in that the radiance depends on the diffuse reflectivity from the whitecap [28]. The radiometric whitecap measurements in the surf zone have shown that the foam-covered areas have a large albedo and reflect about one order of magnitude more than the foam-free areas [29]. Furthermore, the time histories of the passage of the breaking waves indicate sharp increases in reflectance in finite time, followed by an exponential decay.

In general, it is this large difference between the observed reflectivity of the bubbly and nonbubbly surfaces that is exploited to identify the whitecaps. However, most applications do not attempt to discriminate between active breaking and remnant foam, although some attempts have been made using subjective thresholds [30], [31], fractal representations of the intensity signal [32], or an approach based on wave kinematics [23].
A review of the existing methodologies for identifying active wave breaking in optical data shows that most separation methods have been developed for deep water whitecaps and that radiometric studies are rare [8]. Instead, usually the analyzed quantity is an uncalibrated image intensity, henceforth denoted as $I$. Therefore, the discrimination procedure between breaking and nonbreaking image portions is often carried out using relative intensity thresholds, in which pixels with intensities above a given threshold are attributed to the whitecaps. However, the overall image brightness can vary, owing to the changes in the environmental and experimental conditions. The environmental effects include the changes in the sun's zenith and the variations of the cloud coverage, for instance. The experimental effects include the differences in camera looking angles and the changes in camera aperture and shutter speed. As a consequence, no universal rule exists, and the threshold is data set specific and, in some cases, is allowed to vary between images [30], [33]. Recently, a few image processing algorithms have been proposed to reduce the subjectivity in threshold determination and to improve automation [13], [23], [34].

The use of these methods is not straightforward in the surf zone mainly due to the increased levels of breaking frequency and the persistence of the remnant foam, which can last several wave periods. Despite the presence of foam, to date, the main procedure that is used to denote zones of preferential breaking involves the use of time exposures where the contributions of the wave roller and remnant foam are intermingled. Aarninkhof and Ruessink [35] developed a procedure that is used to remove the foam contribution from the mean intensity image (or time exposure) during postprocessing by introducing a model for the decay of the intensity signal, but a separation methodology applicable on a wave-by-wave basis does not yet exist.

The mean and standard deviation of the intensity represent the basic level of characterization given by the lower order moments of the time-varying signal. However, the pdf yields more information. Although the optical pdf has not been previously described for the surf zone, the direct relationship between the observed radiance and the surface slopes for the nonbreaking waves [28] can be exploited. In deep water, the sea surface slope is best described as a Gaussian process whose pdf is described as the product of two Gaussian distributions, which is dependent on the co-wind and cross-wind slopes, respectively [28], [36]. Therefore, it can be expected that the pdf of the radiance would also be Gaussian distributed to first order. It must be noted that this is true only for relatively smooth surfaces (thus small slopes). For the wave fields with steeper slopes, the pdf would show a steep increase at low intensities, followed by a exponential-like decay [28]. However, in the shoaling region, the occurrence of nonlinear, asymmetric, or skewed waves can also lead to the distribution to depart from Gaussian.

The pdf of a broken wave, in turn, will depend on the duration and spatial extent of the breaker and foam coverage. For instance, the deep water whitecap life span exhibits a probability distribution that is almost exponential [28]. This departure from the expected Gaussian distribution of the nonbreaking waves has been used by Mironov and Dulov [23] to discriminate between wave breaking stages in deep water. In the surf zone, 
Aarninkhof and Ruessink [35] introduced a model relating the time series of the optical intensity to the wave period $T$ and a parameter that is related to foam persistence $(\lambda)$. A pdf can be derived for this model

$$
p(I)=\frac{1}{\lambda I}
$$

where $I$ is defined in the interval between a background level $I_{0}$ and the peak intensity $I_{0}+\Delta I_{b}$. It can be seen that the pdf also resembles an exponential decay which is governed by foam persistence. However, this simple model does not take into account the spatial extent of the wave roller, and it also assumes that the brightest point will be located at the wave roller front. Haller and Catalán [8] used high-resolution optical data in the laboratory and showed that the brightest point is colocated with the wave crest, which is followed by a region of approximately constant high intensity values. The effect of those on the pdf will be a departure of the exponential decay, with a secondary peak at high intensity values. Finally, in the surf zone, the signal will usually be the result of a mixture of the breaking and nonbreaking waves. Therefore, the resulting radiance will be the sum of the area-weighted contributions arising from the breaking and nonbreaking areas [37]. In consequence, the pdfs are expected to be a combination of two exponential-like processes at different intensity ranges. However, the intensity threshold at which one process dominates cannot be determined beforehand.

\section{B. Microwave}

At moderate incidence angles $\left(\theta=20^{\circ}-70^{\circ}\right)$, the microwave returns from the ocean surface are explained by Bragg scattering and the composite surface theory (CST) [38], [39], in which the ocean is modeled as a continuum of sloped facets whose extent is small compared to the (long) ocean waves but larger than the Bragg waves. The resulting scattering is then the modulation of the Bragg scattering by the long waves.

However, many observations show scattering signatures that are not consistent with Bragg scattering nor with the CST. These anomalies occur most often for active microwave sensors with low grazing angle (LGA) viewing geometries and include high intensity bursts of backscatter (sea spikes) [24], [25], [40], polarization ratios $(\mathrm{HH} / \mathrm{VV}$, where $\mathrm{HH}$ corresponds to horizontal transmit-horizontal receive and $\mathrm{VV}$ corresponds to vertical transmit-vertical receive) exceeding unity [22], [24], [41], [42], and a broadening of the Doppler spectrum and large Doppler offsets [43], [44]. The breaking waves have been often cited as the source for these anomalies, and the characteristics of the anomalies have been used for breaking wave detection. However, methods that are based on power thresholds lead to large false detection rates [16]. Usually, both breaking and steep unbroken waves are the source of observed sea spikes [25]. The polarization data have shown that the breaking waves exhibit ratios in the vicinity of one, but the polarization ratio alone was not considered to be a reliable discriminator [25]. However, a significant uncertainty still exists as others have assumed that polarization ratios exceeding unity suffice in discriminating the breaking events [42], [45].
In addition to the uncertainty regarding the relationship between the breaking waves and the scattering anomalies, the vast majority of previous studies use observations from deep water where the dynamics of breaking can be different from that in shallow water. In the surf zone, Haller and Lyzenga [17] used $\mathrm{VV}, \mathrm{X}$-band, and colocated video data and defined a spike as an event whose temporal excursion above the mean normalized radar cross section (NRCS) was longer than $0.2 \mathrm{~s}$. They found out that $92 \%$ of the detected events corresponded to the optical breaking signatures identified by an observer. Moreover, their results indicated that the spikes were arising from the scattering from the active breaking region in front of the wave and that the remnant foam was a weaker scattering source, thus opening the possibility to discriminate between these states.

A good understanding of the statistical description of the scattered fields is needed for target detection applications, such as marine surveillance [21]. If the footprints are large and if uniform scatterers are distributed within it, the microwave amplitude will be Rayleigh distributed, and the power will be exponentially distributed [46]. The presence of spikes or highresolution cells induce departures from purely exponential distributions, which have been modeled using compound models, combinations of Weibull distributions, or the K-distribution with varying levels of success [22], [46]-[50]. For instance, at LGA and HH, Trizna et al. [22], [51] found two distinct trends in the distribution, which they cite as evidence of two separate scattering mechanisms.

Here, we shall perform pdf and joint pdf (JPDF) analysis in order to develop a breaking wave identification method. First, we analyze the pdfs of data for each sensor in order to validate the conceptual scattering models presented in this section and their relation with the different stages of wave breaking. Next, we use the JPDF to develop a joint method, in which the different scattering characteristics between sensors and wave breaking stages are exploited to better identify the breaking events.

\section{EXPERIMENTAL DATA}

Nearshore remote sensing observations were collected over a six-week period between April 10 and May 22, 2008, at the U.S. Army Corp of Engineers Field Research Facility (FRF), Duck, NC. In the following, we utilize the FRF coordinate system where the cross-shore coordinate is denoted as $x$ and points offshore, the $y$ axis points roughly $18^{\circ}$ west of north, and $z=0$ correspond to NADV29. For the data analyzed herein, the shoreline was located at approximately $x=90 \mathrm{~m}$ in the FRF coordinate system. The data were collected using three remote sensors. The first one was a single polarization $(\mathrm{HH})$ marine radar (Si-Tex RADARpc-25.9) operating at $9.45 \mathrm{GHz}$ and attached to an independent data acquisition system (Imaging Science Research, Inc.). The radar antenna was mounted atop of a $10-\mathrm{m}$ tower near the north end of the FRF facility $(x=17.4 \mathrm{~m}, y=971.4 \mathrm{~m}$, and $z=13.8 \mathrm{~m}$; see Fig. 1). The marine radar is an active sensor with a $25-\mathrm{kW}$ nominal power and a 9-ft open array antenna that rotates at approximately $44 \mathrm{r} / \mathrm{min}$. Therefore, a point on the surface is sampled every $1.36 \mathrm{~s}(0.73 \mathrm{~Hz})$. A pulse repetition 


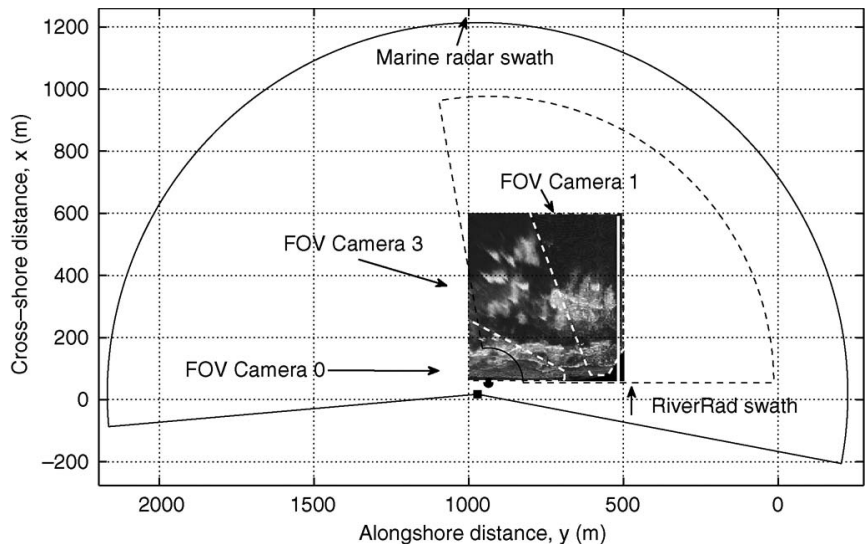

Fig. 1. Field of view of the sensors and sensor location. The circular sectors denote the swath covered by the marine radar (solid line) and RiverRad (dashed line), respectively. As the background, a merged image from the ARGUS III cameras is presented, with the white dashed lines denoting the boundaries between cameras. The vertical white at the $x=516 \mathrm{~m}$ line denotes the location of the FRF pier, and the solid square and circle denotes the location of the marine radar and RiverRad, respectively.

frequency of $2000 \mathrm{~Hz}$ was used along with a pulsewidth of $80 \mathrm{~ns}$, resulting in an intrinsic range resolution of $12 \mathrm{~m}$, although the data acquisition system internally oversamples, yielding a constant working range resolution of $3 \mathrm{~m}$. The horizontal antenna beamwidth is $0.8^{\circ}$, and the vertical beamwidth is about $25^{\circ}$. Data acquisition was designed to average seven received waveforms, which reduced noise and decreased the azimuthal resolution to roughly $2^{\circ}$. Special care was taken to limit the saturation of the received signal by introducing an offset of $-500 \mathrm{mV}$ before processing by using the internal logarithmic amplifier. The recorded signal is then an uncalibrated grayscale intensity index $I_{r}(\theta, r, t)$. The acquisition system records the relative azimuth and time (accurate to $10 \mathrm{~ms}$ ) of each sample, which enables geolocation and synchronization with the other sensors. The maximum recorded ground range was set to $1200 \mathrm{~m}$, and the collections covered a swath of about $200^{\circ}$ under nominal rotation speeds. Thirty-minute collections were recorded at the beginning of each hour throughout the duration of the experiment.

The second remote sensing system was comprised of three optical cameras from the ARGUS III observing station established at the FRF by the Coastal Imaging Laboratory, College of Oceanic and Atmospheric Sciences, Oregon State University (OSU). Further details of this system can be found in [12]. For the purpose of this experiment, a rectangular pixel array was designed, spanning $x=60-600 \mathrm{~m}$ and $y=500-1000 \mathrm{~m}$, with a spatial resolution of $\Delta x=2 \mathrm{~m}$ and $\Delta y=5 \mathrm{~m}$, using cameras 0,3 , and 1, as shown in Fig. 1. The optical (video) pixel intensity data $I(x, y, t)$ were collected simultaneously with the marine radar during daylight hours (seven runs of $31 \mathrm{~min}$ per day) at a sampling rate of $2 \mathrm{~Hz}$.

The third sensor was RiverRad, which is an X-band $(9.36 \mathrm{GHz})$ dual polarization $(\mathrm{HH}, \mathrm{VV})$ coherent radar developed by the Applied Physics Laboratory, University of Washington. It was deployed on the crest of the dune at the north end of the FRF property, at $x=54.4 \mathrm{~m}, y=936.2 \mathrm{~m}$, and $z=10.2 \mathrm{~m}$. The data from this system were less synoptic than the others. The antennas were fixed in the staring mode at $10^{\circ}$ azimuthal increments for 2-min intervals. The total coverage was $80^{\circ}$ in azimuth every $18 \mathrm{~min}$. Further system details can be found in [52]. In the present work, the RiverRad data were only used for cross calibration to convert the marine radar data $I_{r}(\theta, r, t)$ to NRCSs $\sigma_{0}(\theta, r, t)$. This step removes the system and range dependences that are irrelevant to the scattering dynamics, and it is an extension of the procedure presented in [53].

The analysis focuses on three collections labeled as Runs 9, 13 , and 18 , respectively, which occurred during a storm on May 12-16, 2008. As shown in Table I, the environmental conditions show a significant variability in three parameters, the significant wave height, wind speed, and wind direction. The wind direction changed from blowing onshore (upwind relative to the antennas) to offshore directed (downwind) for the last two runs. In addition, the combined effect of wind, tide level, and varying wave heights resulted in varying degrees of foaminess (see Fig. 2), which allows us to evaluate the effect of the remnant foam on the signals.

\section{DATA ANALYSIS}

As stated previously, the data analysis will focus on the marine radar and optical systems. These two systems provide the largest synchronous surf zone coverage, and the duration of both time series allows the use of a large number of points to ensure a statistical significance. The time series is also short enough to ensure that the environmental conditions remained stable. We note that, although the radar scans the field of view in a finite time, for the present purpose, we treat the marine radar image as a snapshot of the surface. This should not be an issue, considering that the time required for the marine radar to scan through the area defined by the pixel array is $t \approx 0.3 \mathrm{~s}$. During this time, at a nominal wave speed of $10 \mathrm{~m} / \mathrm{s}$, waves would travel a distance shorter than a radar resolution cell. In addition, the scan through rate is less than the video sampling rate $(0.5 \mathrm{~s})$.

However, in order to analyze the synchronous instantaneous signals from the two sensors, the differences in sampling rates and spatial resolution need to be removed. This requires at least one of the sensors to be interpolated to a common domain. This is achieved in two steps. The first step involves the interpolation of the time domain of the video signal to the time domain of the marine radar, which is performed on a pixel-by-pixel basis using linear interpolation. The second step is the interpolation of the marine radar data to the higher resolution uniform grid defined by the video pixel array. Additionally, at the boundaries between the field of view of each camera, the differences in camera gain and integration time induce sharp gradients in the optical pixel intensity that is not related with the actual ocean surface. In order to minimize this effect, in the following, we group the data on a camera-by-camera basis, and we do not include the data near the camera boundaries.

In order to analyze the level of correlation between the time series of each sensor, the squared coherence $\gamma^{2}$ was calculated as

$$
\gamma^{2}=\frac{C o^{2}(f)+Q u^{2}(f)}{C_{M R} C_{V}}
$$


TABLE I

Summary of the Wave Conditions Measured at the 8-m Array During the Storm on May 12-16, 2008, For the Three Selected Runs. The Relative (Rel) Directions Are Measured CW With a $0^{\circ}$ Pointing Along the FRF $x$ Axis

\begin{tabular}{ccccccccc}
\hline Run \# & $\begin{array}{c}\text { Marine } \\
\text { Run } \\
\text { (yearday EST) }\end{array}$ & Time & $H_{m o}$ & $T_{p}$ & $\begin{array}{c}\text { Tide } \\
\text { NAVD88 }\end{array}$ & $\begin{array}{c}\text { Wind } \\
\text { Speed } \\
\mathrm{m} / \mathrm{s}\end{array}$ & $\begin{array}{c}\text { Wind } \\
\text { Dir. } \\
\text { TN (rel) }\end{array}$ & $\begin{array}{c}\text { Wave } \\
\text { TN } \\
\text { Dir. (rel) }\end{array}$ \\
\hline 9 & 1341700 & May 13-22:00 & 3.26 & 12.5 & 0.51 & 11.6 & $56(-16)$ & $56(-16)$ \\
13 & 1351300 & May 14-18:00 & 1.97 & 12.5 & 0.26 & 5.4 & $229(157)$ & $64(-8)$ \\
18 & 1361000 & May 15-15:00 & 1.47 & 11.4 & -0.44 & 7.7 & $253(181)$ & $71(-1)$ \\
\hline
\end{tabular}
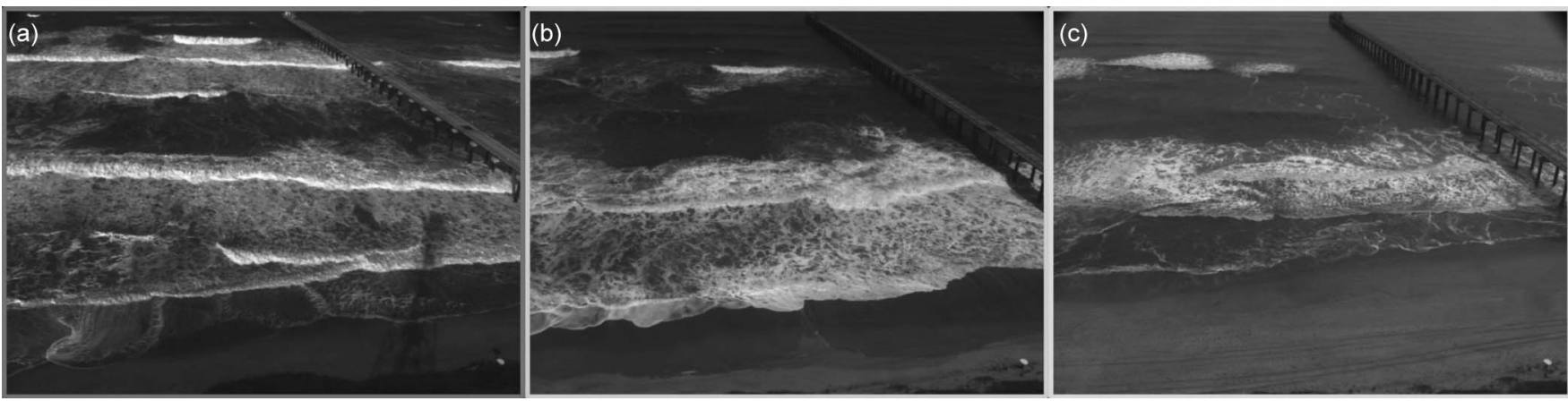

Fig. 2. Video snapshots taken from camera 1 for each of the three selected runs. (a) Run 9. (b) Run 13. (c) Run 18.

for every pixel within the field of view. Here, $C o(f)$ and $Q u(f)$ are the real (cospectrum) and imaginary (quad-spectrum) parts of the cross-spectrum, and $C_{M R}$ and $C_{V}$ are the marine radar and video pixel intensity autospectra [54]. Fig. 3 shows the time exposures from each sensor for each run (video in the top row and marine radar in the middle row). The bottom row shows the median $\gamma^{2}$ value within a window that is $\pm 5 \%$ of the peak frequency of the ocean waves. In the time exposures, the zones of the preferential breaking correspond to areas where the average intensity (or power) is large, shown as brighter areas. As can be seen, the coherence levels are typically large for the majority of the field of view. Therefore, the signals are well correlated. The exceptions are bands of low coherence located on the seaward edges of the areas of preferential breaking. The presence of these bands is due to the intermittent change in the imaging mechanism for the video signal. For the nonbreaking waves, the imaging mechanism is specular reflection, and the local peak in intensity takes place toward the back of the wave where the local incidence angle is a minimum, whereas the wave fronts are dark. Once the waves break, the imaging mechanism in front of the wave changes to diffuse the scattering that is due to the presence of the wave roller, and the signal becomes very bright. For the microwave sensor, the location of the peak power tends to be near the crest of the wave for both breaking and nonbreaking waves. As a consequence, there is a change in the phase between the microwave and video signals as the waves begin to break, which makes the two incoherent at locations where there is an intermittent breaking.

The time exposures show that, during the storm, on average, the waves were breaking near the shoreline and also over an outer bar. In order to differentiate the behavior of the signal between areas of persistent, intermittent, and sporadic breaking, we further divide each camera field of view into four areas, as shown in Fig. 4 and as defined in Table II. Although the characteristics of the signal emanating from each zone will be dependent on the environmental conditions, these zones will remain constant throughout the analysis and will enable the study of the evolution of the signal as the conditions changed.

\section{RESULTS}

\section{A. PDFs}

The aggregate of all of the pixels within each of the zones shown in Fig. 4 produces a joint histogram by counting the frequency of occurrence of a given intensity pair $\left(I, \sigma_{0}\right)$ in the ensemble of samples collected in each run. The integration of the joint histogram along each coordinate axis yields the individual histogram for each data set. Special care is taken to remove the occurrence of signal pairs where there is no marine radar signal (i.e., $I_{r}(\theta, r, t)=0$ ). These are likely points on the water surface that are shadowed by the preceding wave crests (or simply scattering weakly), and they would bias the distributions toward low backscattered power.

The minimum number of usable sample pairs was around 535000 (camera 1, surf zone, and Run 18). The joint and individual histograms were constructed using 25 predefined bins, 11 intensity values wide for video, and $3 \mathrm{~dB}$ wide for marine radar. In the following, the results are presented for the field of view corresponding to camera 1 . The results from the other cameras show a similar behavior unless noted otherwise. In the following, we treat the normalized histograms as a representative of the pdf and JPDF.

1) Video Data: The left column in Fig. 5 shows the pdfs of the video data. In general, the histograms have three different shapes. The first type is the expected peak at low intensity values, e.g., the offshore series for Runs 13 and 18 (circles and asterisks in Fig. 5(a)). These data represent the intensity modulations induced by the wave slope variations of the nonbreaking waves. The resulting signal has a relatively narrow dynamic range, which spans a few bins of the histogram. Also, although the shape is preserved, the means are the offset between runs 

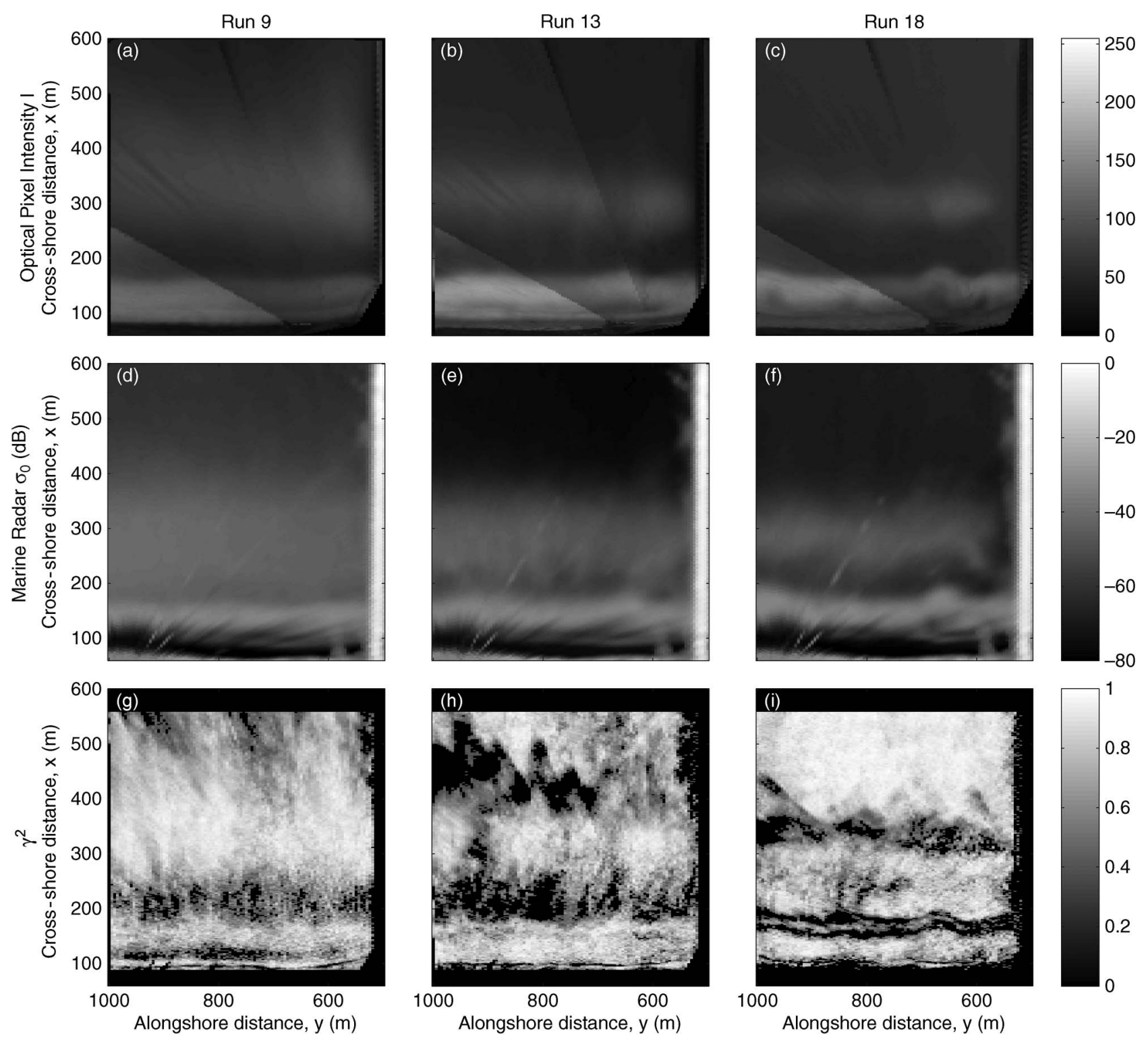

Fig. 3. (Upper row) Thirty-minute time-averaged video images. (Middle row) Thirty-minute time-averaged marine radar images. (Lower row) Median magnitude squared coherence $\left(\gamma^{2}\right)$ within $\pm 5 \%$ of the peak frequency. The columns correspond to different runs.

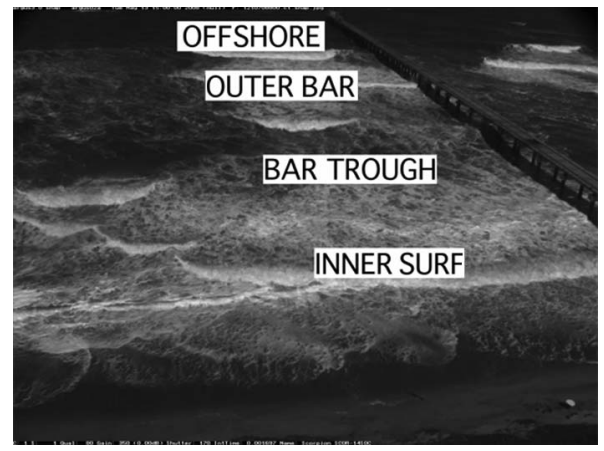

Fig. 4. Definition of the zones within the field of view of camera 1.

mostly owing to the changes in the ambient light, the color of the sea surface, and/or the changes in the camera settings, which were allowed to be adjusted freely between runs depending on the illumination conditions. The wave conditions (wave
TABLE II

Definition of the Zones. The VAlues Represent the Cross-Shore DISTANCE IN THE FRF REFERENCE SYSTEM

\begin{tabular}{lcc}
\hline Identifier & \multicolumn{2}{c}{ Cross-shore limits $(\mathrm{m})$} \\
& $x_{\min }$ & $x_{\max }$ \\
\hline Inner Surf & 100 & 150 \\
Bar Trough & 150 & 250 \\
Outer Bar & 250 & 350 \\
Offshore & 400 & 600 \\
\hline
\end{tabular}

height, period, and, hence, slope) mostly govern the width of the histogram, with a lesser effect on the mean.

The second evident shape is a peak of smaller magnitude at low intensities, followed by an exponentially decaying tail. This kind of behavior would be expected from zones where some wave breaking is taking place with some degree of foam persistency. This can be seen, for instance, in the offshore zone (squares in Fig. 5(a)) where Run 9 differs from the other runs due to intermittent breaking (as shown in Fig. 2). 

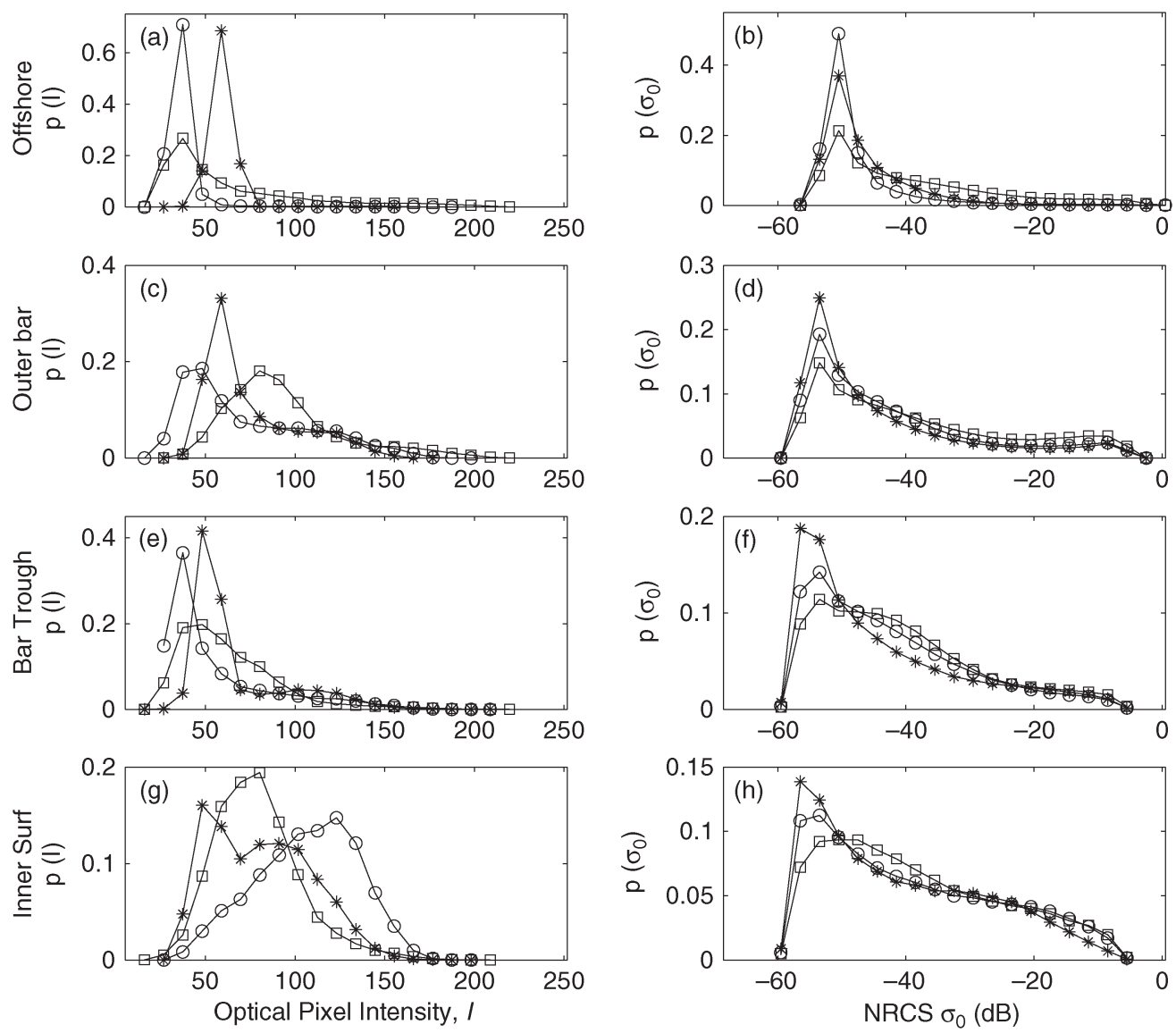

Fig. 5. Histograms of the optical pixel intensity (left) and marine radar NRCS (right) for the data taken within the field of view of camera 1 . Top to bottom correspond to measurements taken in the offshore, outer bar, bar trough, and inner surf zone boxes, respectively. ( $\square$ ) Run 9. (o) Run 13. (*) Run 18 . Note that the vertical axis is different for each panel.

This behavior can also be seen in the outer bar for Runs 9 and 13 (Fig. 5(c); circles and squares, respectively). Finally, the third shape corresponds to conditions where the breaking becomes more frequent and/or foam persistence becomes more pronounced. In which case, the histogram widens, the peak intensity count occurs at larger $I$ values, and the high intensity side of the histogram shows a gently fall off, as can be seen in the inner surf for Runs 9 and 13 (Fig. 5(g); circles and squares).

2) Marine Radar: As shown in the right column of Fig. 5, the majority of the curves for the marine radar show a relatively constant shape, characterized by a sharp peak at a low backscattered power (usually about -55 to $-50 \mathrm{~dB}$ ), followed by an exponential decay, both of which are consistent with the scattering according to the CST model. The magnitude of the peak and the tail contribution show a dependence on the environmental and wave parameters, such as wind-induced roughness or wave breaking. For instance, the effect of wave breaking can be observed in the transition from the high peaks and low tails for Run 18 to the low peaks and high tail values for Run 9 (asterisks and squares in Fig. 5(f)), which represents the relative increase in wave breaking in the trough between those two runs. This is similar to the spatial transition within Run 9 from Fig. 5(d)-(h), as wave breaking steadily increased as the waves propagated onshore.

Of particular interest is the effect of intermittent breaking, for instance, at the outer bar (Fig. 5(d)). In this zone, the histograms for each run are very similar in shape. Each also shows a clear departure from the monotonic decay near $-25 \mathrm{~dB}$, where the histograms instead increase in magnitude, reaching maxima at around $-7 \mathrm{~dB}$, above which they show a decaying trend. Similar results were also found for all runs in the outer bar, bar trough, and inner surf for the field of view of cameras 3 and 0 (not shown) and for the offshore zone in camera 1 (squares in Fig. 5(b)). The increased probability values above the exponential decay at an NRCS greater than $-20 \mathrm{~dB}$ cannot be explained by traditional scattering models. It seems reasonable that these are related to another scattering mechanism. However, a clear identification of the source is not possible with the histogram alone.

\section{B. Joint Histograms}

The results, so far, qualitatively demonstrate how the different water surface types (unbroken waves, active breaking, and foam) manifest themselves in the pdfs. In terms of breaking identification, we know that the challenge is to separate active breaking from remnant foam in the optical data and steep nonbreaking waves from active breaking in the radar data. In order to overcome the limitations of independently using either sensor, we will pursue a sensor fusion approach. We hypothesize that the information from both sensors can be combined in the JPDF (or, in this case, the joint histogram) 

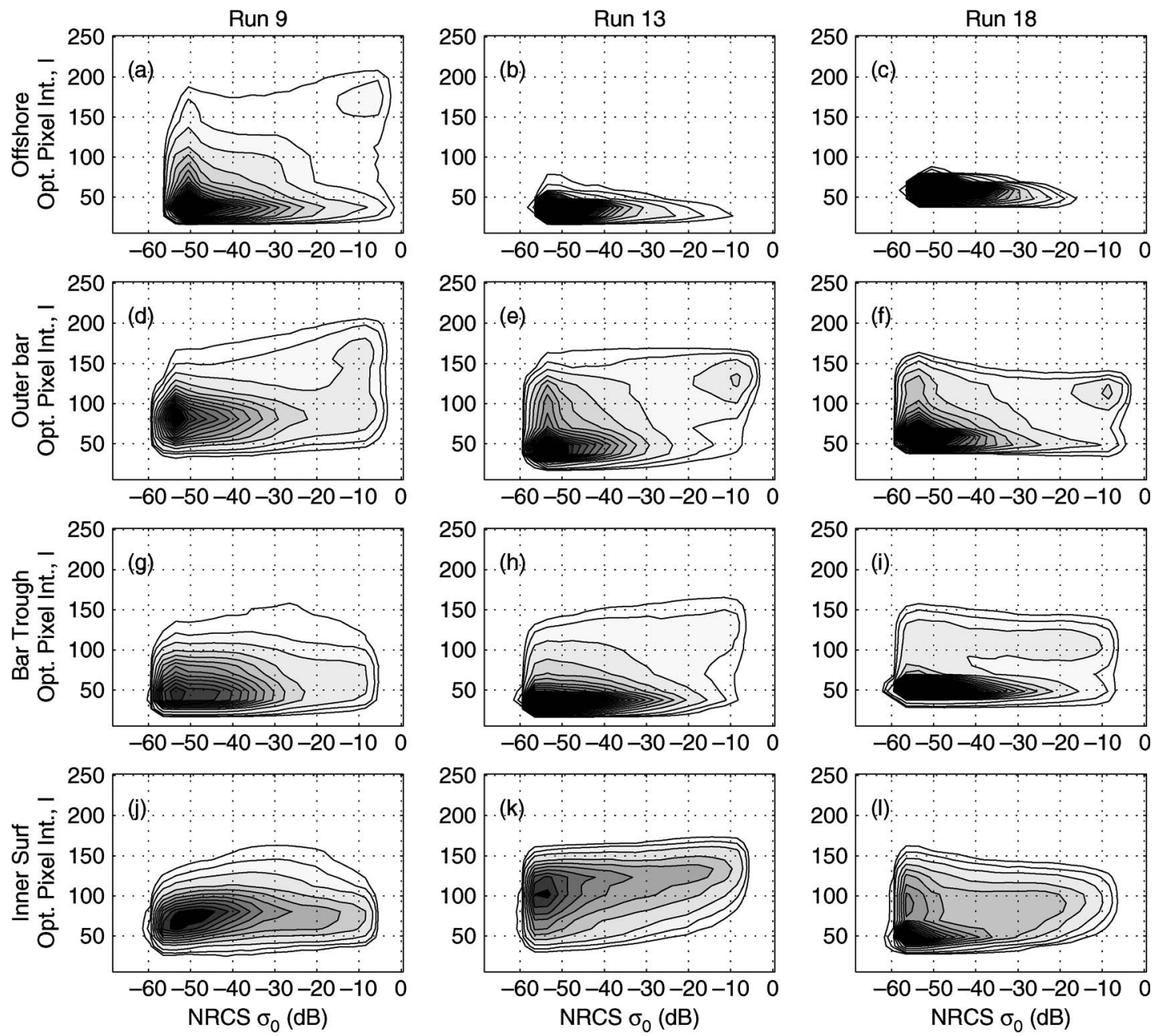

Fig. 6. Joint histograms for the video (camera 1) and marine radar. The columns correspond to Runs 9, 13, and 18, respectively. The rows correspond to zones according to Fig. 4.

in order to better identify the active breaking events and to distinguish them from both the remnant foam and the steep nonbreaking waves.

Fig. 6 shows the JPDFs for all zones in each of the three runs. It can be seen that, typically, the peak of the JPDF occurs at relatively low video intensities and low backscattered powers. We expect the data in this area of the JPDF to arise from the nonbreaking waves, and this is confirmed by the fact that most of the data in panels Fig. 6(b) and (c) (offshore and nonbreaking conditions) are confined to this area. In those panels, the JPDF concentrates along a ridge covering a narrow band of optical intensities (vertical axis, typically between $I=20-70$ ) and a wide range of NRCS levels (horizontal axis). This is due to the slope modulations from the unbroken waves inducing a large dynamic range in the NRCS signal, in accordance with the CST [55], but a relatively narrow dynamic range in the optical signal, which is due only to specular slope modulations [26]. This narrow ridge is also prominent in the data from the bar trough for the decaying wave conditions (Fig. 6(h) and (i)). In addition, it can be seen that the strongest radar returns correspond to the weakest optical intensities (e.g., Fig. 6(c)) from the (optically dark) steep front faces of the waves.
There is also a secondary ridge that is fairly prominent in many cases (e.g., Fig. 6(a), (e), and (f)). This is a vertical ridge spanning a wide range of optical intensity bins ( $I$ up to 150 ) but a relatively narrow range in NRCS (typically -55 to $-50 \mathrm{~dB}$ ). This ridge is indicative of the remnant foam which does not scatter strongly at X-band, but it is optically bright [17].

There is also an evidence in the JPDFs of a secondary peak of lesser magnitude at relatively large optical intensities and large backscattered powers. This local peak is most evident in Fig. 6(a), (d), and (e) for $I>100-125$ and NRCS $>$ $-20 \mathrm{~dB}$. It is totally absent in the nonbreaking conditions (Fig. 6(b) and (c)). Based on our existing understanding, this peak should be the signature of the active wave breaking appearing bright in both sensors. While the peak seems to be consistently backscattering more than $-20 \mathrm{~dB}$, the intensity magnitudes appear to decrease some from Fig. 6(a)-(f) (discussed further in the following). Nevertheless, the JPDF method effectively separates out this portion of the data. For example, the secondary peak in Fig. 6(a) is well separated from the main peak of the nonbreaking waves in the lower left quadrant. 


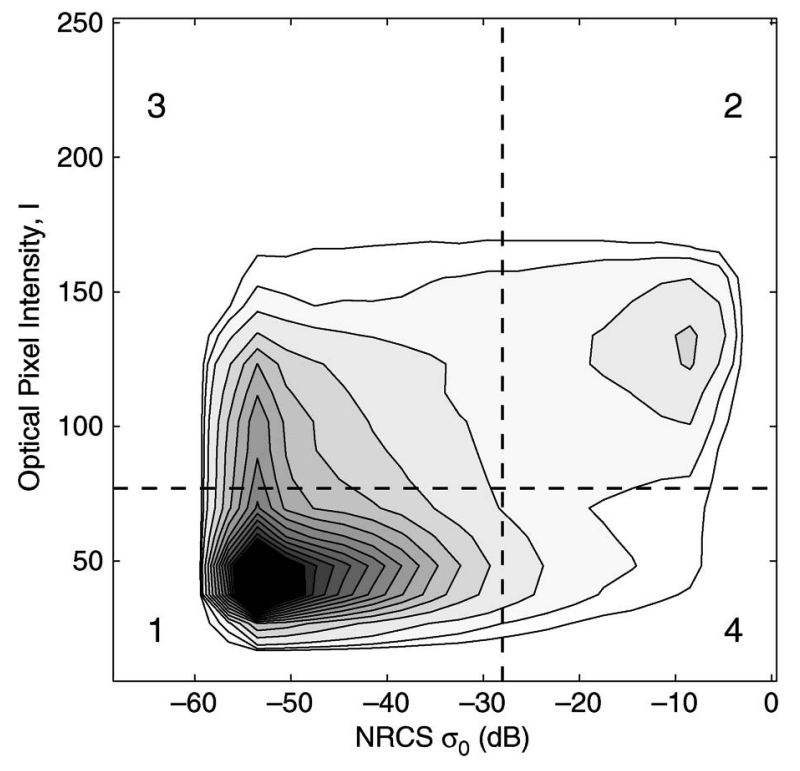

Fig. 7. Characterization of the JPDF in four main regions. The dashed lines denote the discrimination thresholds defined in (3).

\section{Identification of Breaking Events}

The JPDF suggests that, by using simultaneous data from both sensors, the discrimination between different stages of the breaking process is feasible. In particular, it is possible to identify four distinct regions in the JPDF that are associated with different stages of wave breaking in the surf zone. These are shown in Fig. 7. Region 1 corresponds to low optical intensities and low backscattered power (thus the nonbreaking waves). Region 2 corresponds to large optical pixel intensities and large backscattered power due to active breaking waves. Region 3 corresponds to large optical intensities at relatively low backscattered power due to remnant foam. The fourth region corresponds to low optical intensities and large returned power, corresponding to steep waves.

The discrimination procedure is conceptually simple in the sense that, given a suitable selection of the threshold lines shown in Fig. 7, it is straightforward to differentiate between regions. Consequently, the following rules are defined.

Nonbreaking (region 1):

$$
I(x, y, t)<I^{t} \quad \sigma_{0}(x, y, t)<\sigma_{0}^{t} .
$$

Breaking (region 2):

$$
I(x, y, t) \geq I^{t} \quad \sigma_{0}(x, y, t) \geq \sigma_{0}^{t} .
$$

Foam (region 3):

$$
I(x, y, t) \geq I^{t} \quad \sigma_{0}(x, y, t)<\sigma_{0}^{t} .
$$

Steep waves (region 4):

$$
I(x, y, t)<I^{t} \quad \sigma_{0}(x, y, t) \geq \sigma_{0}^{t}
$$

where $I^{t}$ denotes the optical intensity threshold and $\sigma_{0}^{t}$ is the power threshold (in decibels) for the marine radar record.
However, the threshold values still need to be determined, and there may be some variability due to the environmental conditions. A clear example of this is the aforementioned peak in region 2 shown in Fig. 6(d)-(f), which exhibits a steady decrease in optical intensity values. The decrease is most likely a direct result of the change in sun position for the different runs, as the collection times were 6 P.M., 2 P.M., and 11 A.M. local time, respectively. Hence, the sun was moving from a position behind the cameras to a position in front (the cloud cover data were not recorded). Consequently, we first approach the problem in an ad hoc manner using visual inspection to iteratively select the threshold values that appear to provide the best detection rate on a run-by-run basis.

There is really no absolute measure of how well the separation method works. Instead, we must rely on a visual qualitative comparison by overlaying the extracted water surface types onto the video image time series. We recognize that an automated detection algorithm that still relies on qualitative measures of ground truth may seem to be of limited value. Therefore, to clarify our purposes here, clearly, using the "eye of the beholder" as a breaking detection method is highly impractical for large data sets. In addition, the very high resolution data needed for the human (visual) detection of the breaking place heavy demands on data storage and bandwidth capabilities. Hence, here, we have pursued a detection method that is based on thresholds that are initially verified by visual means but, ideally, with limited dependence on the environmental conditions. After these tests, further verification will come from the comparison of the derived breaking wave data with the expected results based on the surf zone dynamics, similar to what was done by Haller and Catalán [8].

Figs. 8 and 9 show the examples of the performance of the joint method of detection for Runs 9 and 18, respectively (the results for Run 13 were similar), where random snapshots (taken from the 30-min-long series) of each sensor are overlaid with spatial contours based on the rules for water surface type. The contours that outline the breaking appear to well correspond with what we consider to be the active breaking region, which travels on the wave fronts. This can be seen, for instance, on the large breaking event spanning $y=520-630 \mathrm{~m}$ and at $x=350 \mathrm{~m}$ in Fig. 8(d). The foam patches also appear to be clearly identified, e.g., the large foamy area centered at $x=$ $300 \mathrm{~m}$ in Fig. 9(d). In the same panel, the detection of the very steep faces is highlighted with the event at $y=640-720 \mathrm{~m}$ and $x=370 \mathrm{~m}$. Moreover, the joint method improves detection especially under conditions when the roller fronts are not so easily discernible from the remnant foam by looking at the video image alone, e.g., the event at $y=670 \mathrm{~m}$ and $x=280 \mathrm{~m}$ in Fig. 9(d). Although in these figures only a snapshot is shown, the visual validation of these and other events (such as the breaking wave identified at $y=720 \mathrm{~m}$ and $x=500 \mathrm{~m}$ in Fig. 8(d)) used the full optical time history of the waves as they shoal and break.

Table III shows the final selected thresholds and some related statistics determined using camera 1. Encouragingly, the table shows that the best performing $I^{t}$ and $\sigma_{0}^{t}$ values are essentially constant between the three runs. Clearly, the optical threshold must be affected by the illumination conditions (e.g., no 

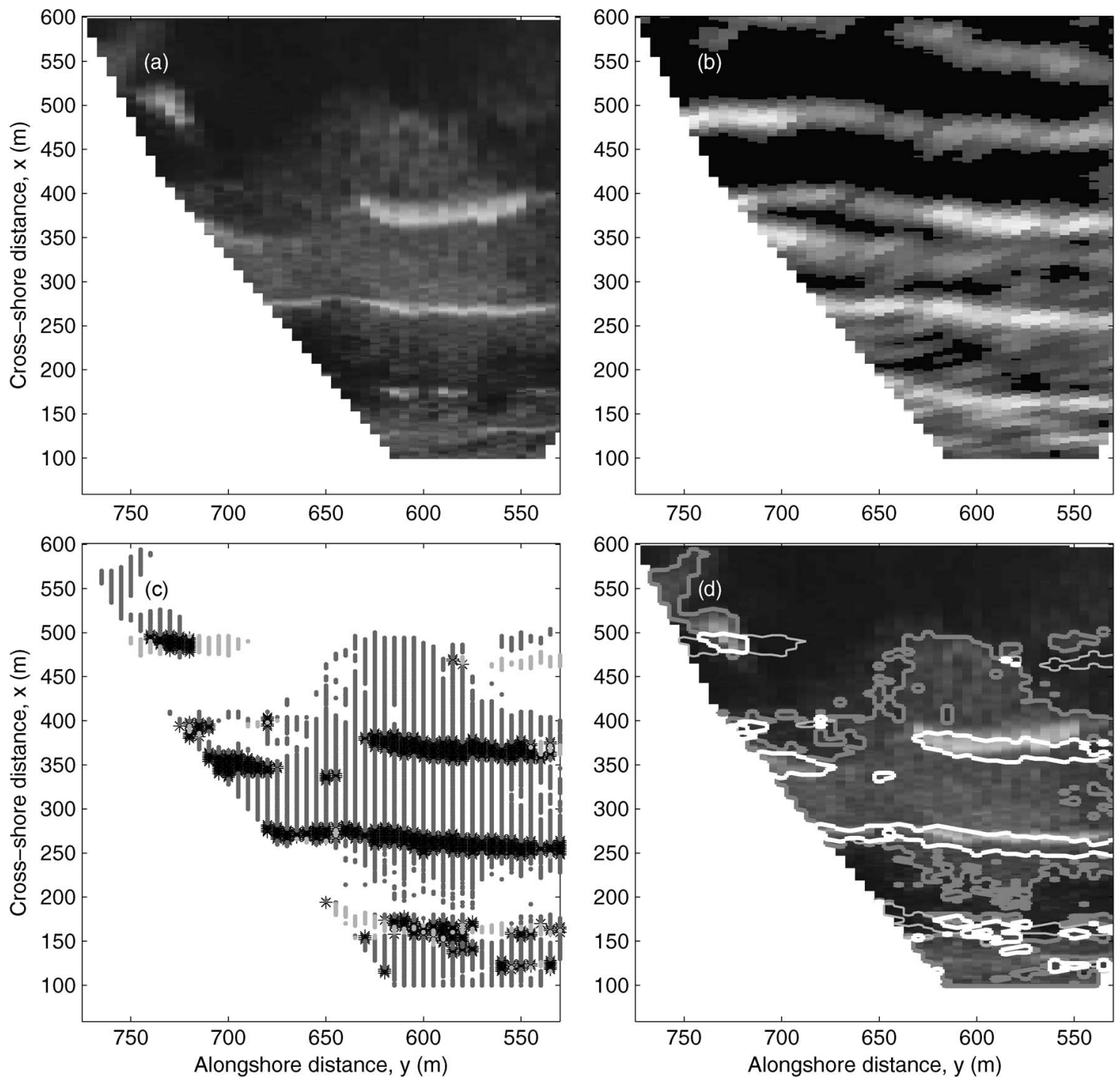

Fig. 8. Combined breaking detection example using camera 1 (Run 9). (a) Video snapshot. (b) Marine radar snapshot. (c) Discrimination results. The black markers denote breaking, the gray markers denote the remnant foam, and the light gray markers denote the steep waves. (d) Discrimination results overlaid as contours over the video snapshot. The white lines denote the breaking events, the dark gray lines denote the remnant foam, and the thin light gray lines denote the steep waves.

threshold exists for the nighttime data), and it shows some variability. However, the cameras have their own automated gain and shutter adjustments, which generally account for preventing saturation and limiting the variability in the detection threshold. Finally, it has to be noted that the selected optical thresholds were found to be $I^{t}=2 / 3 * \bar{I}(x, y)_{\max }$, where $\bar{I}(x, y)_{\max }$ is the spatial maximum over the whole field of view of the time exposure image, suggesting a rule for application to other data.

On the other hand, there was no variation needed in the NRCS threshold for breaking detection partly because the gain setting on the marine radar remains fixed. This suggests a nondependence on the environmental conditions and, possibly, a standard value. However, testing against a larger data set is required. Nevertheless, the suggested threshold value falls within the range of the backscattered powers associated with the departure of the microwave pdf from the exponentially decaying tail.
Next, in order to demonstrate the improvement in detection that the JPDF method provides in comparison to the single sensor methods, the sensitivity of the detection skill to the thresholds $I^{t}$ and $\sigma_{0}^{t}$ is independently tested for each sensor. For the optical record, the thresholds are chosen at selected ratios that are relative to the maximum of the time exposure due to the apparent rule found from the trial-and-error procedure. For the marine radar data, a range of NRCS thresholds on the decibel scale and centered on the baseline found in the joint detection tests is chosen.

Fig. 10 shows the results for the optical records, where each colored area demarcates the region being identified as breaking waves when using a given threshold. Increasing the threshold values yields a better agreement with the visual signature of breaking (see Fig. 8(a) for reference). For Run 9 (Fig. 10(a)), the best agreement is obtained for $I^{t}=1.5 \bar{I}(x, y)_{\max }$, while the lower thresholds tend to include the remnant foam. However, the skill changes for Run 18 (Fig. 10(b) to be compared 

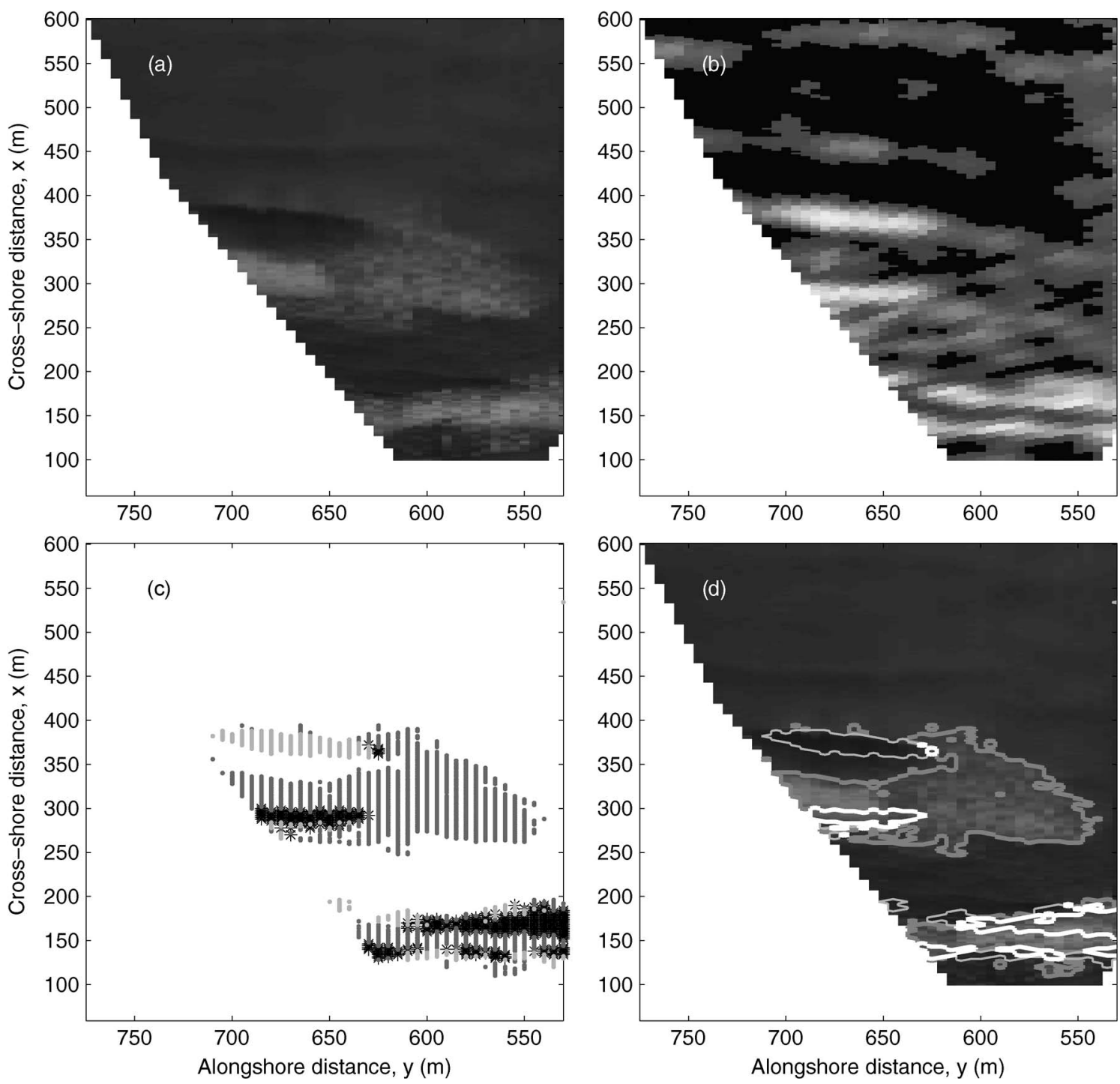

Fig. 9. Combined breaking detection (Run 18). Same key as Fig. 8.

TABLE III

Summary of the Threshold Values for the Combined Breaking Detection Method. The Absolute Maxima "Max()" and the Maxima of the Time Exposure "MaX(-)" Are Provided for Reference. The Video Values Are Given in Grayscale Intensity, AND THE RADAR VAlues ARE IN DeCibels

\begin{tabular}{cccccccc}
\hline Run \# & Video & Video & Video & Video & Radar & Radar & Radar \\
& $I^{t}$ & $\operatorname{Max}(I)$ & $\operatorname{Max}(\bar{I})$ & $I^{t} / \operatorname{Max}(\bar{I})$ & $\sigma_{0}^{t}$ & $\operatorname{Max}\left(\sigma_{0}\right)$ & $\operatorname{Max}\left(\overline{\sigma_{0}}\right)$ \\
\hline 9 & 70 & 219 & 105 & $66 \%$ & -28 & 0 & -17 \\
13 & 77 & 205 & 128 & $60 \%$ & -28 & -1 & -18 \\
18 & 75 & 207 & 119 & $63 \%$ & -28 & -3 & -18 \\
\hline
\end{tabular}

with Fig. 9(a)), where the foam patches tend to be included even at the largest threshold used. These highest thresholds are also nonconservative in that they do not capture the small breaking events and tend to minimize the size of the breaking wave rollers.

For the radar images (Fig. 11), even the use of the largest thresholds tends to overpredict the spatial extent of the roller by including the steepened waves (see $y=640-720 \mathrm{~m}$ and $x=370$ m; Fig. 11(b) compared with Fig. 9(a)). The steep waves that are mistakenly identified as breaking are easier to point out visually than the complementary effect of the foam that is mistakenly identified as breaking. In summary, in Figs. 8-11, it is clear that either sensor acting independently cannot provide a reliable detection with the predefined thresholds in an automated way. On the other hand, the thresholds used successfully in the joint method correspond to the minimum (optical) and mid values (marine radar) tested in Figs. 10 and 11. These thresholds are inherently conservative, and they include the foam and steep waves in the detection when independently used. However, when both sensors are used together, these conservative thresholds appear to work well. 

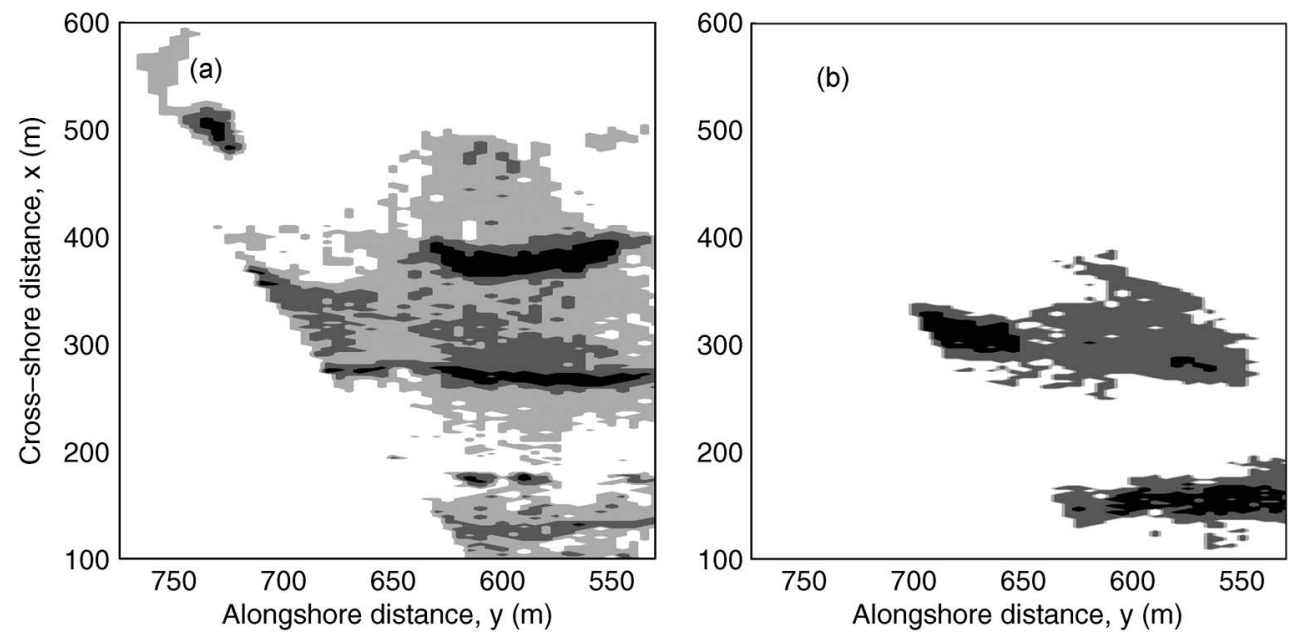

Fig. 10. Sensitivity tests for independent breaking detection using the optical records. (a) Run 9. (b) Run 18. The colored areas denote the events that are identified as breaking using different optical pixel intensity thresholds. The light gray, dark gray, and black areas correspond to the thresholds set at $I^{t}=$ $0.66 \max (\bar{I}), 1.0 \max (\bar{I})$, and $1.5 \max (\bar{I})$, respectively.
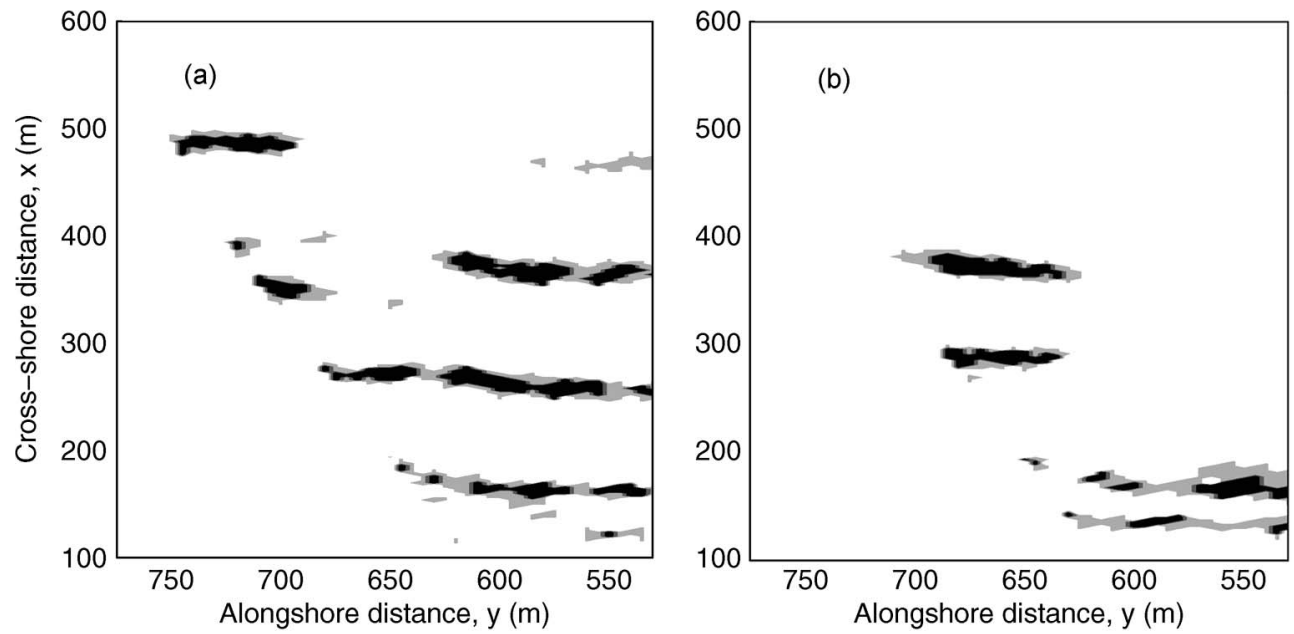

Fig. 11. Sensitivity tests for independent breaking detection using the microwave records. (a) Run 9. (b) Run 18. The colored areas denote the events that are identified as breaking using different NRCS thresholds. The light gray, dark gray, and black areas correspond to the thresholds set at $\sigma_{0}^{t}=-40,-28$, and $-15 \mathrm{~dB}$, respectively.

\section{DISCUSSION}

\section{A. Sample Applications}

The results in the previous section validate the qualitative interpretation of the JPDF and the hypothesis that the joint method can be used for breaking detection. Next, we use the joint method to demonstrate that it can be used to quantify the wave breaking parameters that are important in nearshore processes. For instance, the parametric wave evolution models [2], [3] often rely on a statistical description of the percentage of waves breaking at a given spatial location. Models for this quantity, also known as the fraction of breaking waves $Q_{b}$, have been generally determined and calibrated by visually counting the breaking waves at discrete locations and along a single cross-shore transect [3], [4]. Consequently, the spatial resolution and coverage are often poor. In Fig. 12, the spatial (2-D) distribution of $Q_{b}$ is shown for the three runs, where, for instance, it can be seen that the camera dependences are nicely reduced, as compared to Fig. 3 (upper panels).
Other important parameters are the geometrical properties of the roller. The early work of Duncan [7] showed that, under equilibrium conditions, the dissipation of the energy can be related to the geometry of the wave roller, i.e., the roller cross-sectional area, cross-wave length, and local wave slope. However, a direct measurement of those parameters in the field is a difficult task. Recently, the cross-shore evolution of the roller lengths was linked to roller dissipation using laboratory data [8]. The present approach offers the possibility to extend the technique to field data. For instance, assuming that the waves are propagating normal to the shore, the roller length can be measured as the cross-shore extent of the region identified as a breaking wave at any given alongshore position. As an example, in Fig. 13, the roller lengths obtained from the present data set along a cross-shore transect are shown. These data show a similar (but field scale) cross-shore variation as the laboratory results of Haller and Catalán [8], and if extended in the alongshore direction to obtain the roller areal coverage, they could be used to determine the spatial distribution of the roller dissipation. 


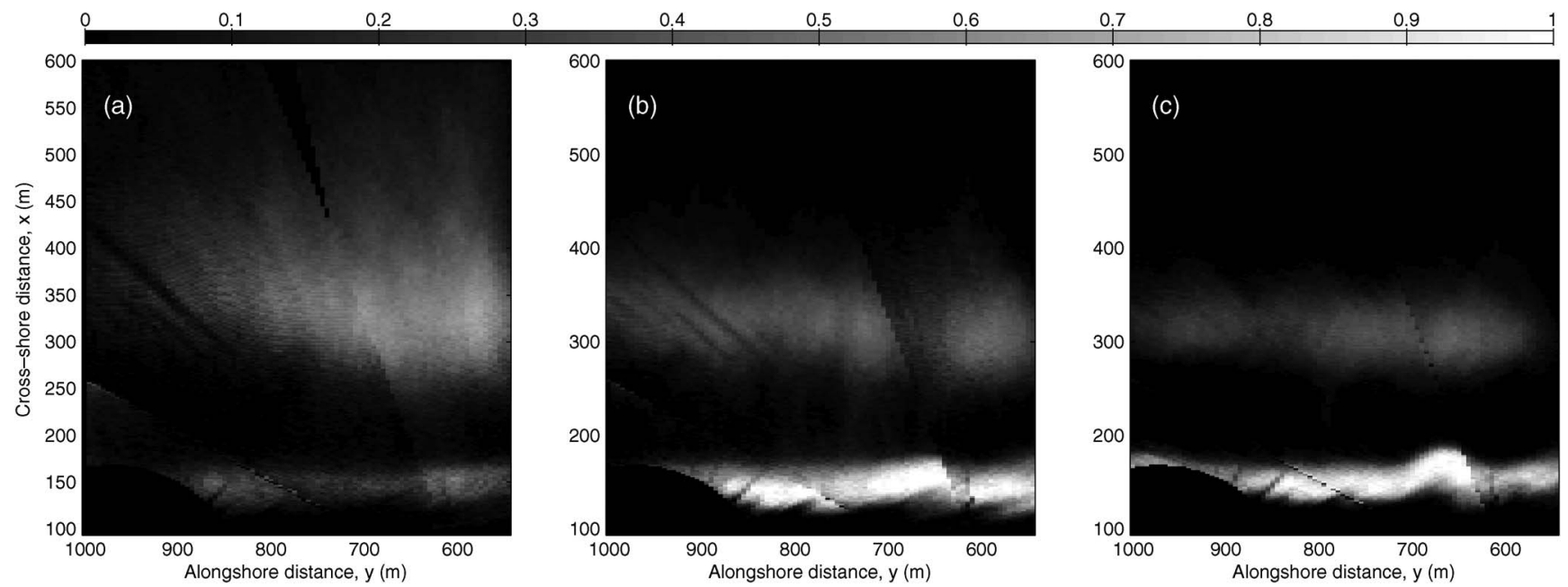

Fig. 12. Computed fraction of the breaking waves for Runs (a) 9, (b) 13, and (c) 18 .
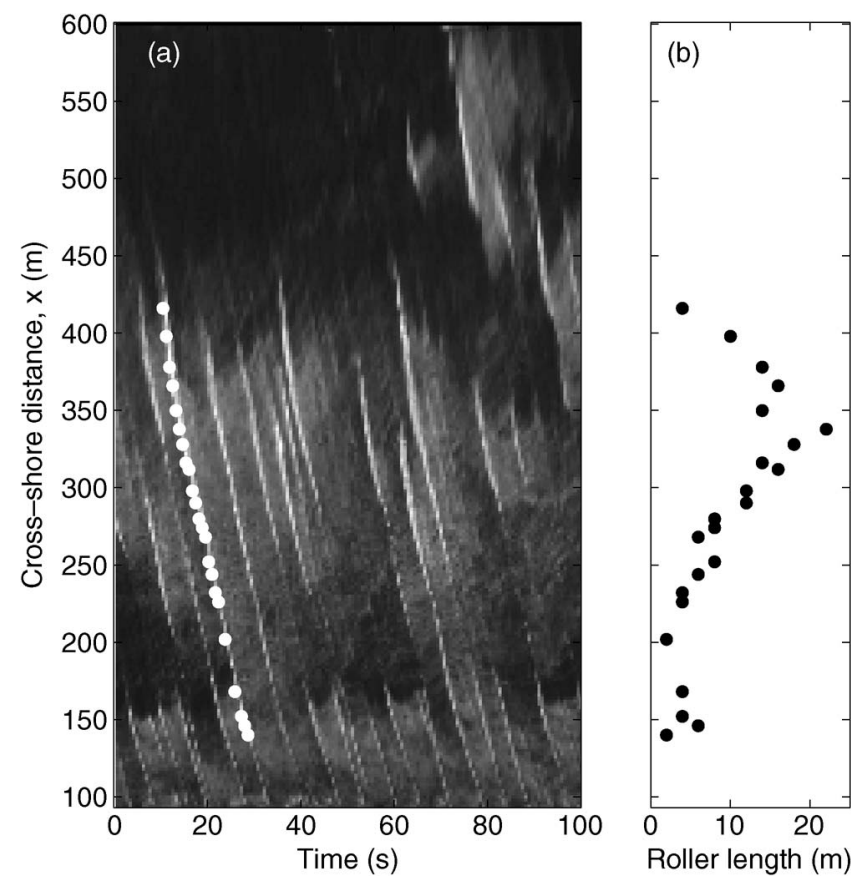

Fig. 13. (a) Time-space map of the optical pixel intensity obtained from a cross-shore transect of Run 9. The white circles correspond to the points along a sample wave trajectory on which the breaking events have been isolated. (b) Measured roller lengths.

\section{B. Sources of Error}

A key to the success of the method is to have the two sensors accurately synchronized and geolocated in order to allow pointto-point comparisons. Potential errors arise from the misregistration of either of the signals. The video is more susceptible to this as the line-of-view registration (as opposed to the time-offlight for the microwave sensor) is affected by finite amplitude waves on which signals from higher vertical elevations than the predefined reference (usually the mean water level) are rectified to the horizontal points further away from the camera. This becomes more pronounced at large range distances (lower grazing angles) and for larger waves. This effect might be strong near the onset of breaking since it is the location of the largest wave amplitude, and the optical signature would be most shifted backwards, relative to the location of the wave crest. Radar misregistration error could arise from changes in (or incorrect determination of) the azimuthal datum for the antenna rotor. Both systems are also subject to synchronization errors in the recorded GPS time stamps. Fortunately, accuracies of $<0.5$ $\mathrm{s}$ are sufficient as the video sampling rate used was $2 \mathrm{~Hz}$ (for the radar, it is $0.73 \mathrm{~Hz}$ ).

The size and orientation of the intrinsic radar resolution cells can be another factor. For instance, we note that the JPDFs do appear less well organized in the inner surf zone (Fig. 6 bottom row). Although the main peak appears due to the nonbreaking phase of the waves, the rest of the data tends to smear out toward the right or the upper right quadrant rather than exhibiting a clear secondary peak. For these cases and location (camera 1 and inner surf), the radar is looking obliquely at the waves. Therefore, the individual radar cells may be encompassing both active breaking and nonbreaking scatterers, thus averaging the returned power toward higher values than when only the nonbreaking waves were present [56]. A more uniform population of the scatterers can be expected when the incoming wave direction is aligned with the radar look direction. This is the case for camera 3, whose pdfs exhibit a very distinct secondary peak (breaking waves) even in the inner surf zone (not shown).

\section{CONCLUSION}

The specific goal of the present work is to develop a methodology for identifying the active breaking events in the surf zone, which minimizes the false alarms that are due to the remnant foam or steep waves. It was found that a joint method using two synchronized remote sensors proved successful in identifying the breaking events on a wave-by-wave basis. The optimal method uses conservative (i.e., high false alarm rate if used independently) joint threshold values that do not vary significantly with the environmental conditions that were tested.

The methodology was developed through an analysis of the individual pdfs determined from the surf zone observations using optical and X-band sensors and through comparisons with the expected distribution functions for each sensor, as found in previous works. The pdfs from the optical sensor were consistent with the existence of two distinct scattering mechanisms. In 
addition, the pdfs from the X-band sensor showed the presence of an inflection point at a large NRCS, which is suggestive of a departure from the Bragg or CST scattering mechanism. This inflection point was located in the range -30 to $-20 \mathrm{~dB}$, and a fixed value of $-28 \mathrm{~dB}$ was used in the detection algorithm. This value proved to be independent of the changes in the ambient environmental conditions during the experiment. Being consistent with the findings of previous research [16], [25], [57], it was found that the strongest backscatter comes from the steep and/or breaking waves. However, unlike most results from a deeper water, it is apparent from the present results that, in the surf zone, the depth-limited breaking waves are the more dominant mechanism.

Finally, the joint method allows several water surface types to be identified, i.e., the active breaking, the remnant foam, and the crests of the steep waves. The method can now be used to quantify important parameters for wave-driven hydrodynamics such as the space-time variability of wave breaking, the fraction of the breaking waves, the wave roller scales, and the wave roller dissipation. In addition, the method can allow a more detailed analysis of the microwave scattering mechanisms and their dependence on the water surface conditions.

\section{ACKNOWLEDGMENT}

The authors would like to thank D. Trizna from the Imaging Science Research, Inc.; the staff of the USACE Field Research Facility; J. Stanley from the Coastal Imaging Laboratory, OSU; and G. Chatham from APL-UW for their support during the field experiment.

\section{REFERENCES}

[1] I. A. Svendsen, Introduction to Nearshore Hydrodynamics. River Edge, NJ: World Sci., 2006, ser. Advanced Series on Ocean Engineering.

[2] J. A. Battjes and J. P. F. M. Janssen, "Energy loss and set-up due to breaking random waves," in Proc. 16th Int. Conf. Coastal Eng., 1978, pp. 569-587.

[3] E. B. Thornton and R. T. Guza, "Transformation of wave height distribution," J. Geophys. Res., vol. 88, no. C10, pp. 5925-5938, 1983.

[4] T. E. Baldock, P. Holmes, S. Bunker, and P. V. Weert, "Cross-shore hydrodynamics within an unsaturated surf zone," Coastal Eng., vol. 34, no. 3/4, pp. 173-196, Sep. 1998.

[5] N. Booij, R. C. Ris, and L. H. Holthuijsen, "A third-generation wave model for coastal regions 1. Model description and validation," J. Geophys. Res., vol. 104, no. C4, pp. 7649-7666, Apr. 1999.

[6] R. Silva, M. Losada, and P. Salles, "Modelling linear wave transformation induced by dissipative structures-Random waves," Ocean Eng., vol. 33, no. 16, pp. 2174-2194, Nov. 2006.

[7] J. Duncan, "An experimental investigation of breaking waves produced by a towed hydrofoil," Proc. R. Soc. Lond. A, Math. Phys. Sci., vol. 377, no. 1770 , pp. 331-348, Jul. 1981.

[8] M. C. Haller and P. A. Catalán, "Remote sensing of wave roller lengths in the laboratory," J. Geophys. Res., vol. 114, no. C7, p. C07 022, Jul. 2009.

[9] M. S. Longuet-Higgins and N. D. Smith, "Measurement of breaking waves by a surface jump meter," J. Geophys. Res., vol. 88, no. C14, pp. 9823-9831, Nov. 1983.

[10] W. K. Melville, M. R. Loewen, F. C. Felizardo, A. T. Jessup, and M. J. Buckingham, "Acoustic and microwave signatures of breaking waves," Nature, vol. 336, no. 6194, pp. 54-56, Nov. 1988.

[11] M. R. Loewen and W. K. Melville, "Microwave backscatter and acoustic radiation of breaking waves," J. Fluid Mech., vol. 224, pp. 601-623, 1991.

[12] R. Holman and J. Stanley, "The history and technical capabilities of ARGUS," Coastal Eng., vol. 54, no. 6/7, pp. 477-491, Jun./Jul. 2007.

[13] A. H. Callaghan and M. White, "Automated processing of sea surface images for the determination of whitecap coverage," J. Atmos. Ocean. Technol., vol. 26, no. 2, pp. 383-394, Feb. 2009.
[14] A. T. Jessup, C. J. Zappa, M. R. Loewen, and V. Hesany, "Infrared remote sensing of breaking waves," Nature, vol. 385 , no. 6611, pp. 52-55, Jan. 1997.

[15] G. O. Marmorino and G. B. Smith, "Bright and dark ocean whitecaps observed in the infrared," Geophys. Res. Lett., vol. 32, no. 11, p. L11 604, Jun. 2005.

[16] A. T. Jessup, W. K. Melville, and W. C. Keller, "Breaking waves affecting microwave backscatter.1. Detection and verification," J. Geophys. Res., vol. 96, no. C11, pp. 20 547-20 559, Nov. 1991.

[17] M. C. Haller and D. R. Lyzenga, "Comparison of radar and video observations of shallow water breaking waves," IEEE Trans. Geosci. Remote Sens., vol. 41, no. 4, pp. 832-844, Apr. 2003.

[18] T. C. Lippmann and R. A. Holman, "Quantification of sandbar morphology: A video technique based on wave dissipation," J. Geophys. Res., vol. 94, no. C1, pp. 995-1011, Jan. 1989.

[19] B. G. Ruessink, P. S. Bell, I. M. J. van Enckevort, and S. G. J. Aarninkhof, "Nearshore bar crest location quantified from time-averaged X-band radar images," Coastal Eng., vol. 45, no. 1, pp. 19-32, Mar. 2002.

[20] J. E. McNinch, "Bar and Swash Imaging Radar BASIR: A mobile $\mathrm{X}$-band radar designed for mapping nearshore sand bars and swashdefined shorelines over large distances," J. Coastal Res., vol. 23, no. 1, pp. 59-74, Jan. 2007.

[21] K. Ward, C. Baker, and S. Watts, "Maritime surveillance radar. I. Radar scattering from the ocean surface," Proc. Inst. Elect. Eng. F-Radar Signal Process., vol. 137, no. 2, pp. 51-62, Apr. 1990.

[22] D. B. Trizna, "Statistics of low grazing angle radar sea scatter for moderate and fully developed ocean waves," IEEE Trans. Antennas Propag., vol. 39, no. 12, pp. 1681-1690, Dec. 1991

[23] A. S. Mironov and V. A. Dulov, "Detection of wave breaking using sea surface video records," Meas. Sci. Technol., vol. 19, no. 1, p. 015 405, Jan. 2008

[24] B. L. Lewis and I. D. Olin, "Experimental study and theoretical model of high-resolution radar backscatter from sea surfaces," Radio Sci., vol. 15, no. 4, pp. 815-828, Jul./Aug. 1980.

[25] Y. Liu, S. Frasier, and R. McIntosh, "Measurement and classification of low grazing angle radar sea spikes," IEEE Trans. Antennas Propag., vol. 46, no. 1, pp. 27-40, Jan. 1998.

[26] B. Jähne, J. Klinke, and S. Waas (1994, Aug.). Imaging of short ocean wind waves: A critical theoretical review. J. Opt. Soc. Amer. A, Opt. Image Sci. [Online]. 11(8), pp. 2197-2209. Available: http://www.opticsinfobase.org/abstract.cfm?URI=josaa-11-8-2197

[27] D. Stilwell, "Directional energy spectra of the sea from photographs," $J$. Geophys. Res., vol. 74, no. 8, pp. 1974-1986, Apr. 1969.

[28] R. E. Walker, Marine Light Field Statistics. New York: Wiley, 1994.

[29] R. Frouin, M. Schwindling, and P.-Y. Deschamps, "Spectral reflectance of sea foam in the visible and near-infrared: In situ measurements and remote sensing implications," J. Geophys. Res., vol. 101, no. C6, pp. $14361-$ 14371, 1996.

[30] D. B. Ross and V. Cardone, "Observations of oceanic whitecaps and their relation to remote measurements of surface wind speed," J. Geophys. Res., vol. 79, no. 3, pp. 444-452, 1974.

[31] E. C. Monahan and D. K. Woolf, "Comments on 'variations of whitecap coverage with wind stress and water temperature'," J. Phys. Oceanogr., vol. 19, no. 5, pp. 706-709, May 1989.

[32] B. R. Kerman and L. Dernier, "Multifractal representation of breaking waves on the ocean surface," J. Geophys. Res., vol. 99, no. C8, pp. 16179 16196, 1994.

[33] C. Lafon, J. Piazzola, P. Forget, O. Le Calve, and S. Despiau, "Analysis of the variations of the whitecap fraction as measured in a coastal zone," Boundary-Layer Meteorol., vol. 111, no. 2, pp. 339-360, May 2004.

[34] Y. Sugihara, H. Tsumori, T. Ohga, H. Yoshioka, and S. Serizawa, "Variation of whitecap coverage with wave-field conditions," J. Mar. Syst., vol. 66, no. 1-4, pp. 47-60, Jun. 2007.

[35] S. Aarninkhof and B. G. Ruessink, "Video observations and model predictions of depth-induced dissipation," IEEE Trans. Geosci. Remote Sens., vol. 42, no. 11, pp. 2612-2622, Nov. 2004.

[36] C. Cox and W. Munk, "Statistics of the sea surface derived from sun glitter," J. Mar. Res., vol. 13, no. 2, pp. 198-227, 1954.

[37] P. Koepke, "Effective reflectance of oceanic whitecaps," Appl. Opt., vol. 23, no. 11, pp. 1816-1824, Jun. 1984.

[38] J. W. Wright, "A new model for sea clutter," IEEE Trans. Antennas Propag., vol. AP-16, no. 2, pp. 217-223, Mar. 1968.

[39] F. G. Bass, I. M. Fuks, A. I. Kalmykov, I. E. Ostrovsky, and A. D. Rosenberg, "Very high frequency radio scattering by a disturbed sea surface: 2, Scattering from an actual sea surface," IEEE Trans. Antennas Propag., vol. AP-16, no. 5, pp. 560-568, Sep. 1968. 
[40] S. J. Frasier, Y. Liu, and R. E. McIntosh, "Space-time properties of radar sea spikes and their relation to wind and wave conditions," J. Geophys. Res., vol. 103, no. C9, pp. 18745-18757, Aug. 1998.

[41] A. T. Jessup, W. C. Keller, and W. K. Melville, "Measurements of sea spikes in microwave backscatter at moderate incidence," J. Geophys. Res., vol. 95, no. C6, pp. 9679-9688, Jun. 1990.

[42] P. A. Hwang, M. A. Sletten, and J. V. Toporkov, "Analysis of radar sea return for breaking wave investigation," J. Geophys. Res., vol. 113, p. $\mathrm{C} 02$ 003, Feb. 2008

[43] P. H. Lee, J. D. Barter, K. L. Beach, C. L. Hindman, B. M. Lake, H. Rungaldier, J. C. Shelton, A. B. Williams, and H. C. Yuen, "X band microwave backscattering from ocean waves," J. Geophys. Res., vol. 100, no. C2, pp. 2591-2611, Feb. 1995.

[44] W. J. Plant, "A model for microwave Doppler sea return at high incidence angles: Bragg scattering from bound, tilted waves," J. Geophys. Res., vol. 102, no. C9, pp. 21 131-21 146, 1997.

[45] P. A. Hwang, M. A. Sletten, and J. V. Toporkov, "Breaking wave contribution to low grazing angle radar backscatter from the ocean surface," $J$. Geophys. Res., vol. 113, p. C09 017, 2008.

[46] B. L. Gotwols and D. R. Thompson, "Ocean microwave backscatter distributions," J. Geophys. Res., vol. 99, no. C5, pp. 9741-9750, May 1994.

[47] B. Gotwols and W. Keller, "Radar backscatter statistics from water waves," in Proc. OCEANS Conf. 'Enginnering in the Ocean Environment', 1990, pp. 240-242.

[48] D. R. Thompson and B. L. Gotwols, "Comparisons of model predictions for radar backscatter amplitude probability density functions with measurements from SAXON," J. Geophys. Res., vol. 99, no. C5, pp. 97259739, May 1994.

[49] D. B. Trizna and D. J. Carlson, "Studies of dual polarized low grazing angle radar sea scatter in nearshore regions," IEEE Trans. Geosci. Remote Sens., vol. 34, no. 3, pp. 747-757, May 1996.

[50] J. V. Toporkov and M. A. Sletten, "Statistical properties of lowgrazing range-resolved sea surface backscatter generated through twodimensional direct numerical simulations," IEEE Trans. Geosci. Remote Sens., vol. 45, no. 5, pp. 1181-1197, May 2007.

[51] D. Trizna, J. P. Hansen, P. Hwang, and J. Wu, "Laboratory studies of sea spikes at low grazing angles," J. Geophys. Res., vol. 96, no. C7, pp. 12 529-12537, Jul. 1991.

[52] W. Plant, W. Keller, and K. Hayes, "Measurement of river surface currents with coherent microwave systems," IEEE Trans. Geosci. Remote Sens., vol. 43, no. 6, pp. 1242-1257, Jun. 2005.

[53] C. Gommenginger, N. Ward, G. Fisher, I. Robinson, and S. Boxall, "Quantitative microwave backscatter measurements from the ocean surface using digital marine radar images," J. Atmos. Ocean. Technol., vol. 17, no. 5, pp. 665-678, May 2000

[54] J. S. Bendat and A. G. Piersol, Random Data Analysis and Measurement Procedures, 2nd revised ed. New York: Wiley, 1986.

[55] W. J. Plant, "Bragg sea scattering of electromagnetic waves from the air/sea interface," in Surface Waves and Fluxes, G. L. Gernaert and W. J. Plant, Eds. Norwell, MA: Kluwer, 1990, ch. 11, pp. 41-108.

[56] H. W. Melief, H. Greidanus, P. van Genderen, and P. Hoogeboom, "Analysis of sea spikes in radar sea clutter data," IEEE Trans. Geosci. Remote Sens., vol. 44, no. 4, pp. 985-993, Apr. 2006.

[57] J. A. Puleo, G. Farquharson, S. J. Frasier, and K. T. Holland, "Comparison of optical and radar measurements of surf and swash zone velocity fields," J. Geophys. Res., vol. 108, no. C3, p. 3100, 2003.

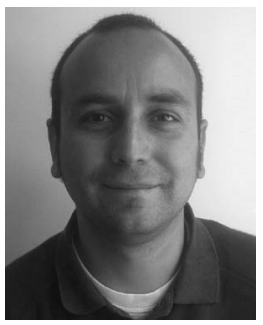

Patricio A. Catalán received the B.S. degree from the Universidad Santa María, Valparaíso, Chile, in 1997 and the M.Oc.E and Ph.D. degrees in civil engineering from Oregon State University, Corvallis, in 2005 and 2008, respectively.

$\mathrm{He}$ is currently an Assistant Professor with the Departmento de Obras Civiles, Universidad Santa María. His research interests include multisensor remote sensing of the nearshore ocean, wave breaking, and nearshore modeling.

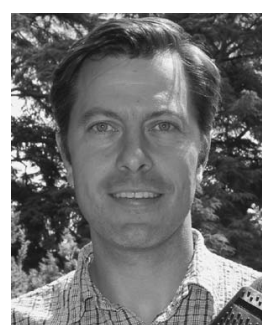

Merrick C. Haller received the B.S. degree from Purdue University, West Lafayette, IN, in 1993 and the M.C.E. and Ph.D. degrees in civil engineering from the University of Delaware, Newark, in 1996 and 1999, respectively.

He presently holds joint appointments as an Associate Professor with the School of Civil and Construction Engineering as well as the College of Oceanic and Atmospheric Sciences, Oregon State University, Corvallis. Previously, he worked as a Research Engineer with Veridian Systems Division and the University of Michigan, Ann Arbor. His present research interest is in nearshore wave transformation and breaking and all that becomes of that

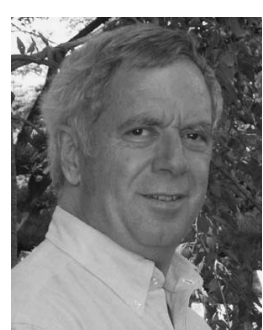

Robert A. Holman received the B.S. degree in mathematics and physics from the Royal Military College of Canada, Kingston, ON, Canada, in 1972 and the Ph.D. degree in physical oceanography from Dalhousie University, Halifax, NS, Canada, in 1979.

He then joined the College of Oceanic and Atmospheric Sciences, Oregon State University, Corvallis, where he is currently a Full Professor. He is also the Secretary of the Navy/Chief of Naval Operations Chair of Oceanography. He is also the Head of the ARGUS Program for nearshore remote sensing. His research interest includes the application of optical remote sensing methods to understand the dynamics of the nearshore ocean.

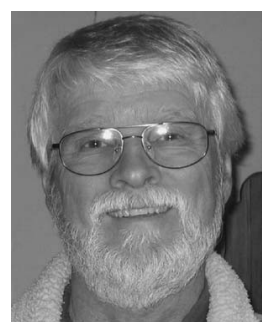

William J. Plant (M'93) received the B.S. degree in physics from Kansas State University, Manhattan, in 1966 and the M.S. and Ph.D. degrees in physics from Purdue University, West Lafayette, IN, in 1968 and 1972 , respectively.

$\mathrm{He}$ is a Principal Research Scientist with the Applied Physics Laboratory, University of Washington, Seattle, where he is the past Chairman. From 1971 to 1973 , he was a National Research Council Postdoctoral Research Associate with the Naval Research Laboratory (NRL), Washington, DC. In 1973, he was with the staff of NRL and remained there until 1988, serving as the Head of the Ocean Measurements Section during the final three years. In 1988, he moved to the Woods Hole Oceanographic Institution, Woods Hole, MA, as a Senior Scientist before coming to the Applied Physics Laboratory in 1991. In 1995, he was a Visiting Scientist with the Max-Planck-Institut fur Meteorologie, Hamburg, Germany, and in 2000, he was a Visiting Scientist with the Rosenstiel School of Marine and Atmospheric Sciences, University of Miami, Coral Gables, FL, where he is an Adjunct Professor with the Division of Applied Marine Physics. He has served as an Associate Editor of the Journal of Geophysical Research and the Journal of Oceanic Engineering. He has authored or coauthored over 80 scientific and technical publications and has coedited two books. The primary objectives of his research are to investigate the microwave scattering from rough water surfaces and to develop algorithms to obtain the geophysical information from such scattering.

Dr. Plant was awarded the Distinguished Technical Achievement Award by the IEEE Oceanic Engineering Society in 1993. In 2003, he was awarded the John Wesley Powell Award by the U.S. Geological Survey. 